\title{
La calidad de vida percibida en pacientes diabéticos tipo ${ }^{2}$
}

\author{
Luz Marina Bautista Rodríguez² \\ Gloria Esperanza Zambrano Plata³
}

Como citar: Bautista Rodríguez LM, Zambrano Plata GE. La calidad de vida percibida en pacientes diabéticos tipo 2. Investig Enferm. Imagen Desarr. 2015;17(1):131-148.

1. Articulo original de investigación. Fecha de recepción: 14 de agosto de 2014. Fecha de aceptación: 30 de septiembre de 2014.

2. Maestría en Enfermería con énfasis en Gerencia en Servicios de Salud. Docente titular, Universidad Francisco de Paula Santander, Cúcuta, Colombia. Correo electrónico: bautrod@gmail.com

3. Maestría en Enfermería con énfasis en Salud Materno Perinatal. Docente asociada, Universidad Francisco de Paula Santander, Cúcuta, Colombia. Correo electrónico: gloriae91 @hotmail.com 


\section{Resumen}

Objetivo: Describir y comparar por sexo la calidad de vida percibida por los adultos con enfermedad diabética tipo 2 , pertenecientes a instituciones de salud del primer nivel de atención. Metodología: Estudio cuantitativo descriptivo, transversal, con una muestra constituida por 287 pacientes que cumplieron los criterios de inclusión y aceptaron participar en el estudio mediante expresión verbal del consentimiento informado. Para recolectar la información se utilizó el cuestionario de Diabetes Quality of Life, que permite conocer la calidad de vida del paciente diabético, mediante la valoración de las subescalas satisfacción con el tratamiento, impacto del tratamiento, efectos sociales y vocacionales, efectos futuros de la enfermedad y bienestar general. Resultados: De los pacientes diabéticos tipo 2 que participaron en el estudio, el $62,3 \%$ tenía una calidad de vida media, respecto a la percepción subjetiva del estado de salud, el 46,7\% consideró tener un estado de salud bueno. En la relación de las puntuaciones promedio obtenidas en cada una de las subescalas, la más afectada es la del impacto en el tratamiento con una puntuación directa promedio de 41,1 y la menos afectada es la preocupación por los efectos futuros de la enfermedad, con 9,9. Conclusión: El estudio mostró que los 287 pacientes diabéticos tipo 2 perciben que la calidad de vida no se afecta en gran medida por la presencia de la enfermedad.

Palabras clave: calidad de vida; diabetes mellitus; estado de salud; bienestar

\section{Perceived Quality of Life in Type 2 Diabetic Patients}

\section{Abstract}

Objective: To describe the quality of life perceived by adults with type 2 diabetes disease health institutions belonging to the first level of care. Methodology: Descriptive, cross-sectional study; with a sample of 287 patients who met the inclusion criteria and agreed to participate in the study by verbal expression of informed consent. Diabetes Questionnaire Quality of Life to find out the quality of life of diabetic patients, by assessing subscales satisfaction with treatment, impact of treatment, social and vocational effects of future effects was used for data collection disease and overall wellness. Results: Of type 2 diabetic patients who participated in the study, $62.3 \%$ have a quality of life media, regarding perceived health status, $46.7 \%$ considered to have a state of good health. The ratio of the average scores on each of the subscales, the most affected is the impact of treatment with a direct scoring average of 41.1 and the least affected is the concern for the future effects of the disease, 9.9. Conclusion: The study shows that the 287 type 2 diabetic patients perceive the quality of life is not affected greatly by the presence of the disease.

Keywords: quality of life; diabetes mellitus; health status; welfare 


\section{Qualidade de vida em pacientes com diabetes mellitus}

\section{Resumo}

Objetivo: Descrever a qualidade de vida percebida por adultos com diabetes tipo 2, que pertencem as instituições de saúde no primeiro nível de atenção. Metodologia: Estudo descritivo, transversal, com uma amostra de 287 pacientes que preencheram os critérios de inclusão e aceitaram participar no estudo de expressão verbal de consentimento informado. Para coletar dados, foi usado o Questionário de Qualidade de Vida Diabetes, que permite descobrir a qualidade de vida dos pacientes diabéticos, avaliando sub-escalas de satisfação com o tratamento, o impacto do tratamento, social e efeitos profissionais de efeitos futuros e bem-estar geral. Resultados: Dos pacientes diabéticos tipo 2 que participaram do estudo, $62,3 \%$ têm uma qualidade de vida media, sobre o estado de saúde percebida, 46,7\% consideram ter um bom estado de saúde. A relação entre as notas médias em cada uma das sub-escalas, a mais afetada é o impacto do tratamento com uma média de pontuação direta de 41,1 e menos afetada é a preocupação com os efeitos futuros da doença 9,9. Conclusão: O estudo mostra que os pacientes com diabetes tipo 2287 perceber a qualidade de vida não é afetada significativamente pela presença da doença.

Palauras chave: qualidade de vida; diabetes mellitus; nível de saúde; bem-estar 


\section{Introducción}

La diabetes mellitus, por su naturaleza crónica, por la severidad de las complicaciones y por los medios que se requieren para su control, se constituye en el momento en una enfermedad altamente costosa. La evidencia acumulada en años recientes demuestra que el diagnóstico temprano y el buen control de la diabetes reducen la progresión de complicaciones crónicas de la enfermedad, como la retinopatía, la nefropatía, la neuropatía y la muerte, y que, a la vez, mejora la calidad de vida de estas personas (1).

En la Declaración de las Américas sobre la Diabetes se plantea que cuando esta enfermedad está mal controlada, puede representar una pesada carga económica para el individuo y la sociedad. Dependiendo del país, la diabetes puede alcanzar entre $5 \%$ y el $14 \%$ de la población; pero es posible promover la salud y prevenir las complicaciones en las personas que la padecen mediante un buen control de la glucemia y modificando los factores de riesgo cardiovascular (2).

El incremento de la diabetes en el mundo es alarmante. De acuerdo con las estadísticas de la Federación Internacional de la Diabetes: "en 1985 había 30 millones de personas diabéticas en el mundo; en 1998, un poco más de una década después, la cifra se incrementó a 143 millones de afectados" (1,3). La diabetes mellitus es un problema de salud pública mundial. Según la Organización Panamericana de la Salud, en el documento Situación de salud en las Américas: indicadores básicos 2006, se estimó para ese año que 35 millones de personas eran diabéticas y se prevé que esa cantidad aumentará a 64 millones para el 2025; así mismo, se considera que en los próximos 10 años las defunciones por enfermedades crónicas aumentarán en un $17 \%$ y, lo que es más alarmante, las defunciones causadas por la diabetes aumentarán en casi el $80 \%$ (4).

En el país, las estadísticas no son diferentes. Según la Asociación Colombiana de Diabetes, se estima que el $7 \%$ de la población colombiana mayor de 30 años de edad que reside en el área urbana tiene diabetes tipo 2 (5). De acuerdo con la Organización Panamericana de la Salud y la Organización Mundial de la Salud, en el informe Situación de salud de las Américas: indicadores básicos 2013, la mortalidad por diabetes para 2011 fue de 18,1 por cada 100.000 habitantes. En Norte de Santander, según el Instituto Departamental de Salud, la diabetes mellitus tipo 2 ocupa el puesto 14, con 74 casos y un porcentaje de 1,22\% (6). En las unidades básicas del Instituto Municipal de Salud de Cúcuta, la diabetes mellitus tipo 2 resulta ser un problema de salud pública muy común que se presenta como causa de consulta tanto en hombres como en mujeres en edades entre 41 a 91 años, al ocupar los cinco primeros puestos de las causas de consulta externa que se registraron en los meses de enero a febrero de 2010.

Esta enfermedad ocasiona padecimientos de larga duración, de carácter no transmisible y degenerativo con etiología incierta, múltiples factores de riesgo, periodos de latencia largos, curso prolongado, fuente no contagiosa, discapacidad o alteración funcional e incurabilidad. Además, impone dos tipos de carga: afecta la productividad de los individuos y su 
capacidad de generar ingresos y origina un mayor consumo de servicios sociales y de salud, generalmente de alto costo (7).

Dado que la diabetes tipo 2 es más frecuente en personas obesas y de nivel socioeconómico bajo, "representa una carga social importante que se manifiesta tanto en los sistemas de servicios de salud como en la calidad de vida de las personas diabéticas y sus familiares" (8). Así mismo, el carácter de cronicidad de la diabetes en los adultos mayores es un factor determinante en la calidad de vida de estas personas, razón por la cual en el ámbito internacional se ha trabajado en estrategias que orienten el desarrollo de programas para mejorar la atención y calidad de vida de personas con esta patología.

En América Latina y el Caribe existen diferentes modelos de atención a las personas con enfermedades crónicas. El Modelo de Atención a Crónicos en un esfuerzo por mejorar la calidad de la atención al paciente con diabetes mellitus, específicamente el utilizado en México, el cual incluye acciones para reducir la mortalidad por enfermedades crónicas, como la creación de unidades médicas de especialidad (Uneme), las cuales basan su acción en combinar la prevención con el tratamiento eficaz de los afectados (9).

La Organización Panamericana de la Salud y la Organización Mundial de la Salud, por medio de la Red Panamericana del proyecto Conjunto de Acciones para la Reducción Multifactorial de las Enfermedades no Transmisibles (CARMEN), apoyan las actividades de prevención de factores de riesgo para la salud en las Américas: "La meta fundamental de esta estrategia es prevenir y reducir la carga de Enfermedades Crónicas No Transmisibles y sus factores de riesgo en las Américas" (10).

En Colombia, mediante la norma técnica para la prevención de enfermedad crónica y mantenimiento de la salud en el individuo sano mayor de 45 años se brinda atención del adulto mayor a esta edad. Esta atención se define:

[...] como un conjunto de actividades, procedimientos e intervenciones, mediante los cuales se logra la detección temprana y el diagnóstico oportuno de las patologías prevalentes propias de este grupo poblacional, con el fin de ofrecer alternativas para el control de los factores de riesgo, el tratamiento o la rehabilitación, lo cual permite cumplir el objetivo de la norma de prevenir y detectar precozmente las enfermedades más prevalentes en este grupo de edad. (11)

En las Américas, la diabetes es un grave problema de salud pública y de alto costo que aqueja a todos, sin distinguir edades o niveles socioeconómicos. Hay millones de personas que no saben que tienen la enfermedad y otras tantas que, a pesar de que se les ha diagnosticado, no reciben el tratamiento apropiado. Se subestima la repercusión de la diabetes en las sociedades y las personas. Las personas con diabetes, cuya enfermedad está mal controlada, tienen un riesgo mayor y una incidencia elevada de 
ataques cardiacos, accidentes cerebrovasculares, ceguera, insuficiencia renal, amputación de piernas y muerte prematura. La diabetes no solo acorta la vida productiva, sino que tiene serias repercusiones en la calidad de vida del enfermo y la de su familia (12).

La calidad de vida relacionada con la salud tiene una creciente importancia como estimador del resultado de los programas e intervenciones de los profesionales de la salud en el ámbito sanitario-asistencial. Su uso ha sido denominado como una medida centrada en el paciente, la cual, por un lado, mide la opinión de estos respecto a su propia salud en las dimensiones física, psicológica y social y, por el otro, ha sido tomada como una de las variables finales para evaluar la efectividad de las actuaciones médicas del personal de salud y, por ende, de los programas de las instituciones de salud (13).

A medida que las enfermedades crónicas han aumentado su prevalencia de forma lenta pero progresiva, ha aumentado el interés por la calidad de vida del enfermo y por los múltiples factores biopsicosociales que inciden en ella. "La gran cantidad de aspectos psicosociales que hacen parte de los cuidados en las enfermedades crónicas surge de las respuestas individuales de los pacientes, las familias, los amigos y la sociedad, frente al diagnóstico y al pronóstico. Cualquiera que sea el resultado de la enfermedad, el objetivo es mejorar la calidad de vida" (14).

El concepto de salud está fundamentado "en un marco biosicológico, socioeconómico y cultural, que tiene en cuenta los valores positivos y negativos que afectan la vida, la función social y la percepción; desde esta perspectiva el concepto de salud es de naturaleza dinámica y multifuncional, donde se da relevancia a la calidad de vida" (15). La calidad de vida es un concepto relacionado con el bienestar físico-social y depende de la satisfacción de las necesidades humanas.

La enfermedad crónica es un acontecimiento único para el individuo, la familia y el cuidador familiar, porque ocasiona alteraciones de tipo físico, psicológico, social y espiritual en la salud y en la calidad de vida de los individuos que la sobrellevan durante gran parte de sus vidas. De acuerdo con lo planteado por Jean Watson, citada en el artículo de Lina María Vargas Escobar, "el cuidado de enfermería durante las situaciones crónicas implica acciones transpersonales e intersubjetivas de protección y apoyo para individuos cuidadores y familiares, que les permita encontrar sentido y significado a la enfermedad, su existencia, el sufrimiento y el dolor" (16).

La labor de enfermería, en el caso de las personas que viven en situaciones de cronicidad, se centra en el cuidado de su experiencia de vida con la enfermedad, y para poder realizar este cuidado se "precisa el reconocimiento del mundo que rodea a familiares, individuos y cuidadores, explorando en ellos sus patrones de vida y su conciencia en torno a la situación de cronicidad" (16). En la medida en que enfermería permita este reconocimiento en su cuidado, favorecerá el crecimiento y el poder de los individuos ante la situación de cronicidad (17). 
Jean Watson considera que la prestación de cuidados es un aspecto central de la enfermería, y lo refiere como el "centro unificador de la práctica. La enfermera debe realizar diversas intervenciones, entre ellas la promoción de la salud de las personas a través de acciones preventivas tales como el reconocimiento de las habilidades de adaptación frente a los daños, fomentar la enseñanza-aprendizaje interpersonal y la provisión de apoyo situacional" (18). Estos lineamientos fundamentan el desarrollo de acciones de educación como herramienta para que las personas se informen, mejoren sus estilos de vida y adopten conductas saludables que contribuyan a la calidad de vida.

La enfermería tiene un papel importante en relación con el mantenimiento, la promoción de la salud y la calidad de vida de los individuos y de las comunidades. Hablar y pensar en la salud como la promoción de la calidad de vida requiere "no solo pensar en la solución de los problemas relacionados con su recuperación, sino en actividades de integración con el orden público, capaces de transformar los entornos/espacios en locus saludables y armoniosos para vivir" (19). En este punto, cabe señalar que las cuestiones relacionadas con la calidad de vida han adquirido importancia de muchas maneras en los últimos años, particularmente con respecto a su evaluación o medición, ya sea individual o colectivamente.

Para enfermería es fundamental participar en el cuidado de la salud y la vida de las personas con padecimientos crónico-degenerativos, donde se contribuya a mejorar la calidad de vida mediante información, educación y atención directa de personas afectadas por la enfermedad o sus familias.

\section{Objetivo}

Describir y comparar por sexo la calidad de vida percibida por los adultos con enfermedad diabética tipo 2, pertenecientes a instituciones de salud del primer nivel de atención.

\section{Metodología}

Se realizó un estudio de tipo cuantitativo descriptivo transversal. La población corresponde a 390 pacientes que asisten a consulta o control, y la muestra estuvo constituida por 287 pacientes de instituciones de salud del primer nivel de atención. Esta se seleccionó mediante muestreo probabilístico aleatorio simple, realizando un listado general de los pacientes que asisten a consulta o control y después seleccionándolos al azar, de acuerdo con el tamaño de la muestra establecida.

Para la recolección de información se utilizó el Cuestionario de Calidad de Vida en Diabetes (DQOL), por ser un instrumento breve y sencillo que puede ser autoadministrado, que permite su aplicación en los consultorios de consulta externa. E1 DQOL también permite conocer la satisfacción, el impacto y la preocupación que tiene el paciente diabético. Ello, porque "es un instrumento innovador desarrollado originalmente para su uso en el 
control de la diabetes y sus complicaciones (DCCT) en la década de 1980. Este instrumento mide la calidad de vida de personas con diabetes" (20).

El DQOL está compuesto por 43 ítems que forman 4 dimensiones: satisfacción con el tratamiento (15 ítems), impacto del tratamiento (17 items), preocupación social/vocacional (7 items) y preocupación relativa a los efectos futuros de la diabetes ( 4 items). Cada item tiene 5 opciones de respuesta de tipo Likert que puntúa de 1 a 5 . En la subescala de satisfacción, las respuestas a cada ítem oscilan entre muy satisfecho (1 punto) a nada satisfecho (5 puntos). En las otras tres subescalas, el rango de las respuestas es el siguiente: nunca ( 1 punto) a siempre (5 puntos). La valoración del tipo de calidad de vida global se obtiene a partirla escala establecida según las categorías de alta: 90-0, media: 183-91 y baja: 230-184.

E1 DQOL se caracteriza por tener una adecuada consistencia interna, con alfas de Cronbach de 0,47 a 0,92, y altos coeficientes de estabilidad temporal, que van desde 0,78 a 0,92. En lo que respecta a la validez de constructo, se ha probado que el DQOL se relaciona de manera moderada y consistente con medidas de sintomas psicológicos, bienestar y ajuste a enfermedad y con medidas genéricas de calidad de vida (20-23).

Dentro de los principios éticos que se garantizaron en este estudio están: no maleficencia, justicia, beneficencia, autonomía y confidencialidad. Por tratarse de una investigación con bajo riesgo, el consentimiento libre e informado se obtuvo de forma verbal (artículo 16 de la Resolución 08430 de 1993), previa información de la justificación, objetivos, procedimiento para recolectar la información, molestias y beneficios de la investigación. Se garantizó que en cualquier momento que los pacientes diabéticos decidieran suspender la entrevista o la participación en el estudio lo podían hacer sin que esto representara desmejora de la atención que recibe en la institución.

La información recolectada fue revisada y verificada para identificar el diligenciamiento completo de cada instrumento. Seguidamente, se diseñó y alimentó la base de datos en Excel®. El paquete estadístico utilizado para el análisis de la muestra fue PASW Statistics, versión 18 (SPSS). Dicho análisis estuvo basado en medidas de frecuencia para variables nominales y ordinales, y medidas de tendencia central y dispersión para el caso de variables cuantitativas. Se estableció la diferencia de medias para los resultados obtenidos por subescalas, entre las variables sexo y estado civil de los pacientes, con la realización de la prueba T de Student para muestras independientes, bajo un nivel de significancia de 0,05.

\section{Resultados}

La muestra estuvo integrada por 287 pacientes con diabetes mellitus tipo 2 . El rango de edad se encontró entre los 50 y los 80 años y más. Los grupos etáreos de mayor representatividad son el de 50-54 años (23,3\%) y el de 70-74 años (23,0\%). La edad promedio en el grupo de pacientes fue de 65,9 años, con un coeficiente de variación de 10,1 años. El 42,5\% de 
los encuestados se encontró en una edad inferior o igual a 64 años. Con relación al sexo, el 54,7\% representó el género femenino, y el 45,3\%, el género masculino.

E1 59,9\% de los pacientes vive con pareja actualmente (casados o en unión libre); mientras que el $40,1 \%$ no tiene pareja en la actualidad (solteros, divorciados o viudos). E1 $60 \%$ de los pacientes contó con un vínculo afectivo estable, situación a favor para estos individuos, por poseer un apoyo familiar cercano en relación con el tratamiento que deben seguir para mantener un estado de salud óptimo.

La escolaridad promedio del grupo de pacientes diabéticos que participó en el estudio es de 4,64 con un coeficiente de variación de 3,3 años. La situación social y económica ha sido condicionante en el desarrollo educativo de las personas: el $30 \%$ contaba con estudios de primaria incompleta, y el 51,6\%, con estudios de primaria completa.

Al relacionar la calidad de vida y el estado civil, se puede observar en la tabla 1 cómo el comportamiento de cada una de las dimensiones de calidad varía para los pacientes con pareja y sin pareja; además, existe una calidad de vida media para ambos tipos de pacientes, que se diferencia de manera mínima en los que tienen pareja.

TABla 1. Comparación del deterioro de la calidad de vida por estado civil

\begin{tabular}{|c|c|c|c|c|c|c|}
\hline Escala & Estado civil & $\mathbf{n}$ & Media & $\begin{array}{l}\text { Desvia- } \\
\text { ción típica }\end{array}$ & $\begin{array}{l}\text { Error ti- } \\
\text { pico de } \\
\text { la media }\end{array}$ & $\begin{array}{l}\text { Valor } \\
\text { de p }\end{array}$ \\
\hline \multirow{2}{*}{$\begin{array}{l}\text { Satisfacción con el } \\
\text { tratamiento }\end{array}$} & Sin pareja & 115 & 35,40 & 7,445 & 0,694 & \multirow{2}{*}{0,00} \\
\hline & Con pareja & 172 & 32,61 & 8,543 & 0,651 & \\
\hline \multirow{2}{*}{$\begin{array}{l}\text { Impacto en el trata- } \\
\text { miento }\end{array}$} & Sin pareja & 115 & 41,99 & 6,621 & 0,617 & \multirow{2}{*}{0,09} \\
\hline & Con pareja & 172 & 40,51 & 7,927 & 0,604 & \\
\hline \multirow{2}{*}{$\begin{array}{l}\text { Preocupación por } \\
\text { aspectos sociales y } \\
\text { vocacionales }\end{array}$} & Sin pareja & 115 & 10,32 & 3,305 & 0,308 & \multirow[b]{2}{*}{0,24} \\
\hline & Con pareja & 172 & 10,78 & 3,055 & 0,233 & \\
\hline \multirow{2}{*}{$\begin{array}{l}\text { Preocupación acerca } \\
\text { de los efectos futu- } \\
\text { ros de la enfermedad }\end{array}$} & Sin pareja & 115 & 10,10 & 2,103 & 0,196 & \multirow[b]{2}{*}{0,15} \\
\hline & Con pareja & 172 & 9,73 & 2,163 & 0,165 & \\
\hline \multirow{2}{*}{ Calidad de vida } & Sin pareja & 115 & 97,81 & 14,258 & 1,330 & \multirow{2}{*}{0,03} \\
\hline & Con pareja & 172 & 93,63 & 16,977 & 1,294 & \\
\hline
\end{tabular}

Fuente: DQOL. 
Se encontró que la satisfacción del tratamiento, según las respuestas obtenidas (tabla 2), en el 37,9\% de los pacientes fue muy satisfecho con la apariencia de su cuerpo, y el 43,2\% fue muy satisfecho con su vida social y amistades; mientras que entre el 4,1\% y el 5,2\% estuvo insatisfecho con la flexibilidad de su dieta y con el tiempo libre.

TABLA 2. Distribución según la dimensión satisfacción con el tratamiento

\begin{tabular}{|c|c|c|c|c|c|c|}
\hline Aspecto/valoración & $\begin{array}{c}\text { Muy } \\
\text { satisfe- } \\
\text { cho }\end{array}$ & $\begin{array}{c}\text { Más o } \\
\text { menos } \\
\text { satisfe- } \\
\text { cho }\end{array}$ & $\begin{array}{c}\text { Ningu- } \\
\text { no }\end{array}$ & $\begin{array}{c}\text { Más o } \\
\text { menos } \\
\text { insatis- } \\
\text { fecho }\end{array}$ & $\begin{array}{c}\text { Muy } \\
\text { insatis- } \\
\text { fecho }\end{array}$ & Total \\
\hline Tiempo manejo enfermedad & 79 & 130 & 43 & 33 & 2 & 287 \\
\hline Tiempo chequeos & 60 & 170 & 18 & 36 & 3 & 287 \\
\hline Tratamiento actual & 79 & 148 & 16 & 37 & 7 & 287 \\
\hline Tiempo determinar azúcar & 38 & 177 & 20 & 49 & 3 & 287 \\
\hline Flexibilidad dieta & 36 & 104 & 37 & 95 & 15 & 287 \\
\hline Carga enfermedad familia & 22 & 102 & 117 & 40 & 6 & 287 \\
\hline Conocimiento enfermedad & 44 & 193 & 16 & 27 & 7 & 287 \\
\hline Horas de sueño & 70 & 132 & 14 & 66 & 5 & 287 \\
\hline Vida social y amistades & 124 & 116 & 17 & 24 & 6 & 287 \\
\hline Vida sexual & 58 & 86 & 122 & 17 & 4 & 287 \\
\hline Trabajo y actividades casa & 72 & 120 & 58 & 38 & 7 & 295 \\
\hline Apariencia de su cuerpo & 109 & 137 & 10 & 27 & 4 & 287 \\
\hline Tiempo ejercicio & 66 & 134 & 26 & 53 & 8 & 287 \\
\hline Tiempo libre & 88 & 107 & 32 & 48 & 12 & 287 \\
\hline Vida general & 63 & 177 & 17 & 22 & 8 & 287 \\
\hline
\end{tabular}

Fuente: DQOL.

En el impacto del tratamiento se encontró que el 76,6\% de los pacientes no ocultó a su familia o amigos si el tratamiento farmacológico produce efectos; el 74,5\% expresó que la presencia de la enfermedad no le impidió conducir o manejar otros equipos, y el 68,9\% no presentó dolor asociado con la presencia de diabetes, como se puede observar en la tabla 3. 
TABla 3. Distribución según la dimensión impacto con el tratamiento

\begin{tabular}{|c|c|c|c|c|c|c|}
\hline Aspecto/valoración & Nunca & $\begin{array}{l}\text { Muy } \\
\text { poco }\end{array}$ & $\begin{array}{c}\text { Algu- } \\
\text { nas } \\
\text { veces }\end{array}$ & $\begin{array}{c}\text { Con } \\
\text { fre- } \\
\text { cuen- } \\
\text { cia }\end{array}$ & $\begin{array}{l}\text { Todo el } \\
\text { tiempo }\end{array}$ & Total \\
\hline Dolor asociado al tratamiento & 198 & 63 & 21 & 4 & 1 & 287 \\
\hline Pena por la enfermedad & 132 & 100 & 50 & 5 & 0 & 287 \\
\hline Baja de azúcar & 56 & 99 & 108 & 22 & 2 & 287 \\
\hline Enfermedad física & 4 & 77 & 145 & 48 & 13 & 287 \\
\hline Interferencia enfermedad familia & 45 & 130 & 102 & 10 & 0 & 287 \\
\hline Dormir mal & 39 & 148 & 80 & 20 & 0 & 287 \\
\hline Limitación social y amistades & 109 & 124 & 42 & 10 & 2 & 287 \\
\hline Sentirse bien con él mismo & 12 & 32 & 50 & 146 & 47 & 287 \\
\hline Restricción por dieta & 74 & 90 & 79 & 28 & 16 & 287 \\
\hline Interferencia vida sexual & 166 & 93 & 22 & 6 & 0 & 287 \\
\hline Interferencia manejar & 214 & 44 & 24 & 4 & 1 & 287 \\
\hline Interferencia ejercicio & 155 & 93 & 29 & 9 & 1 & 287 \\
\hline Inasistencia trabajo, escuela & 149 & 99 & 34 & 4 & 1 & 287 \\
\hline Explicar significado diabetes & 57 & 108 & 95 & 24 & 3 & 287 \\
\hline Interrupción tiempo libre & 69 & 151 & 58 & 9 & 0 & 287 \\
\hline Hablar enfermedad & 42 & 127 & 98 & 20 & 0 & 287 \\
\hline Molestia por enfermedad & 169 & 74 & 29 & 14 & 1 & 287 \\
\hline Frecuencia al baño & 42 & 112 & 111 & 16 & 6 & 287 \\
\hline Comer alimentos prohibidos & 131 & 101 & 41 & 13 & 1 & 287 \\
\hline Ocultar reacciones insulina & 220 & 30 & 32 & 5 & 0 & 287 \\
\hline
\end{tabular}

Fuente: DQOL

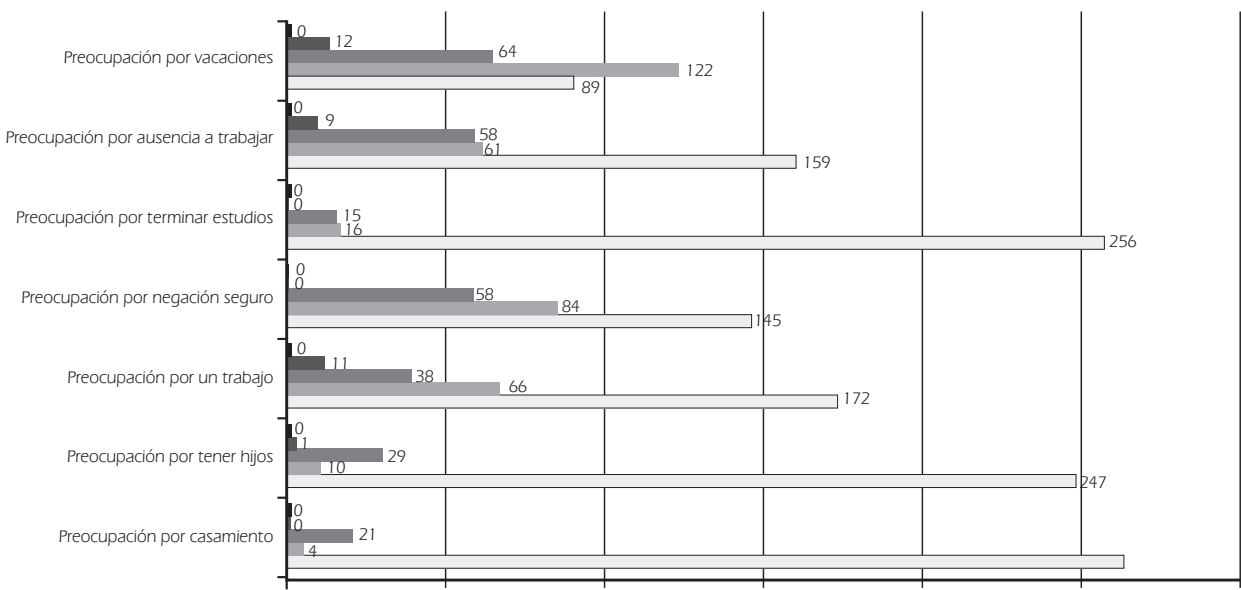

Figura 1. Distribución según la dimensión preocupación por aspectos sociales y vocacionales

Fuente: DQOL 


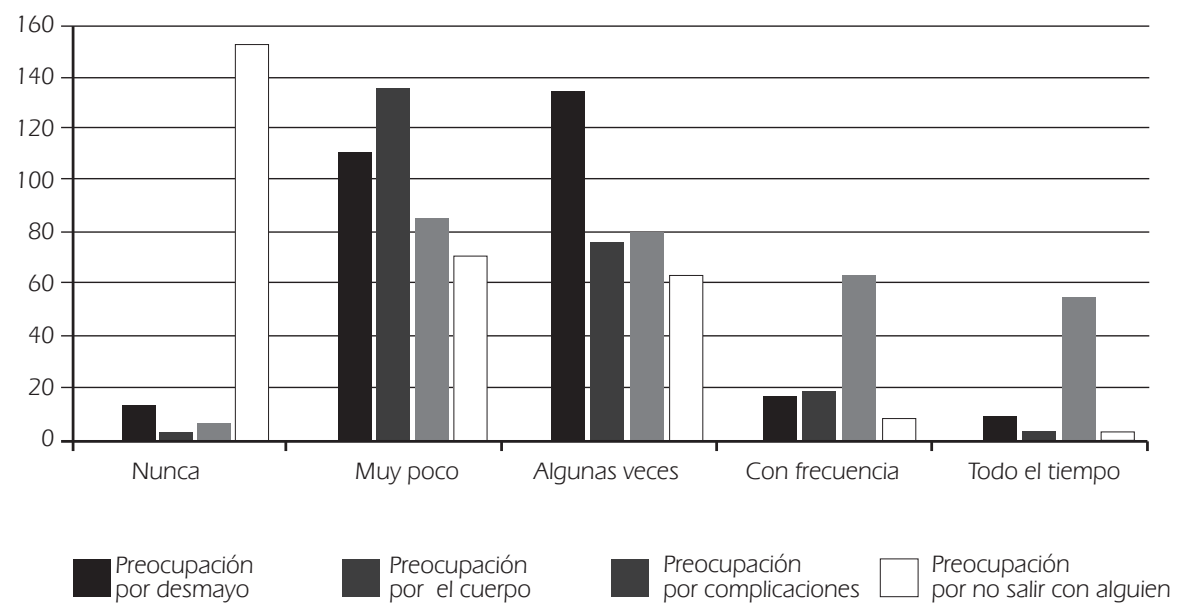

Figura 2. Distribución según la dimensión preocupación acerca de los efectos futuros de la enfermedad

Fuente: DQOL

La preocupación por aspectos sociales y vocacionales hace referencia a los efectos sociales, laborales y económicos que la presencia de la enfermedad puede hacer en la persona; en la figura 1 se diferencian los diferentes aspectos relacionados con esta subvariable y se encontró que lo que nunca preocupó a los pacientes diabéticos está relacionado con la pareja (91,2\%) y los hijos (86\%). Algunas veces se preocupó por si podrían realizar un viaje o ir de vacaciones $(22,2 \%)$, si la presencia de la enfermedad puede generar incapacidad y tendrian que faltar al trabajo (20,2\%) y la negación de la atención en salud (20,2\%).

Con relación a los efectos futuros que pueda generar la enfermedad, en la figura 2 se observa que al 51,5\% no le preocupó que otras personas no se relacionen con él por la presencia de la enfermedad y al 45,6\% algunas veces se preocupó por que se pueda desmayar.

Los pacientes diabéticos, respecto a la percepción subjetiva del estado de salud, consideraron que en el 3,5\% de los casos es excelente y en el $46,7 \%$ es bueno; mientras que el $45,6 \%$ fue regular y el 4,2 \% fue pobre.

Los resultados de la investigación muestran que el 62,3\% de los pacientes manifiestan tener una calidad de vida media, el 37,6\% expresa poseer una calidad de vida alta y ningún paciente tiene calidad de vida baja. Los pacientes con calidad de vida media presentaron persistencia de sintomas propios de la diabetes, como la poliuria, la presencia de complicaciones cardiovasculares específicamente, las dificultades para adoptar una dieta balanceada y la valoración subjetiva del estado de salud por sentirse enfermo. 


\section{Discusión}

Los resultados obtenidos en las características sociodemográficas de los pacientes diabéticos tipo 2 del estudio muestran predominio del sexo femenino y el rango de edad de 50 a 54 años. Estos hallazgos son semejantes a otros estudios realizados con este tipo de pacientes, de acuerdo con Robles y cols. (23), Antuña y Hirsch (24), De los Ríos y cols. (13) y González y Rivas (25). Lo anterior permite ver cómo esta patología inicia en personas relativamente jóvenes en etapa productiva, situación que evidencia de qué manera los factores de riesgo propios de los estilos de vida están llevando a manifestaciones de esta enfermedad en adultos jóvenes y, sobre todo, en mujeres, quienes epidemiológicamente se ven más afectadas por esta situación de salud. Gualdrón (26) encontró que el predominio del género femenino hace a estas personas más propensas a comprometerse en acciones de promoción de la salud.

Se logró determinar que no existen diferencias significativas en la calidad de vida con respecto al género. Las puntuaciones promedio obtenidas para cada de una de las subescalas satisfacción con el tratamiento (hombres: 33,53; mujeres: 33,89), impacto con el tratamiento (hombres: 41,06; mujeres: 41,14), preocupación por aspectos sociales y vocacionales (hombres: 10,43; mujeres: 10,73) y preocupación por los efectos futuros de la enfermedad (hombres: 130; mujeres: 157), así como la media general con un valor de $p$ de 0,45 de la calidad de vida (hombres: 94,53 y mujeres: $95,94)$ son similares para ambos sexos.

El contar con el apoyo de una pareja es un factor común presente en otras investigaciones realizadas en diabéticos tipo 2, como los estudios de Robles y cols. (23), De los Ríos y cols. (13) y Salazar y cols. (27). Por otra parte, al relacionar los resultados obtenidos en el estudio en cada una de las subescalas con el estado civil, se encontró que la dimensión de calidad de vida "satisfacción con el tratamiento" presentó diferencias significativas. Los pacientes sin pareja obtuvieron una mayor puntuación promedio que aquellos pacientes con pareja, lo cual significa un mayor deterioro en la calidad de vida con respecto a esta subescala, en personas solteras, divorciadas o separadas. En general, existen diferencias significativas en la calidad de vida de los pacientes con respecto al estado civil, pues se observó mayor puntuación promedio en pacientes sin pareja, que en aquellos con una relación estable. Meissner, citado Valadez y cols., señaló que:

[...] la enfermedad en el ser humano tiene dos componentes, uno patológico y uno ecológico, en este último, la familia y los amigos constituyen el contexto social más significativo y duradero. Un individuo con diabetes se encuentra todos los días ante sus propias necesidades, dirigiéndose casi siempre a su familia o personas cercanas en busca de ayuda y consejo para solucionar sus problemas de salud; la respuesta que obtenga, influirá positiva o negativamente sobre su conducta terapéutica y, por lo tanto, en el control de la diabetes. (9) 
Otra variable relevante es la escolaridad, pues tener un bajo nivel afecta la poca información que estos pacientes manejan acerca de su enfermedad, prevención de complicaciones y medidas terapéuticas (28). El nivel de escolaridad en pacientes con enfermedades crónicas como la diabetes influye para obtener información y asumir un rol positivo en el autocuidado que debe iniciar y mantener desde el momento en que conoce el diagnóstico. Como lo plantean Quirantez y cols. (2), el descontrol de la enfermedad es consecuencia directa de una deficiente o ausente educación del paciente con diabetes y de sus familiares; de ahí se deriva una baja percepción de riesgo y la no observancia de medidas elementales para la prevención de complicaciones.

Con relación a la calidad de vida, la subescala más afectada es la del impacto en el tratamiento, con un promedio de 41,1, y la menos afectada es la preocupación por los efectos futuros de la enfermedad. Estos resultados difieren de los estudios realizados por Cárdenas y cols. (29), los cuales encontraron que la subescala más afectada fue satisfacción con el tratamiento, y la menos afectada fue impacto de la diabetes. De los Ríos y cols. (13) encontraron que el área más afectada es la psicosocial, donde el $60 \%$ de los sujetos no mostró deterioro, y en $15 \%$ fue severo ( $p<0,003)$. González y Rivas (25) encontraron que la subescala más afectada es la relacionada con el control de la diabetes y ansiedad-preocupación. Gervas (30) halló que el aspecto que ha obtenido la valoración más desfavorable es la satisfacción con el tratamiento.

La calidad de vida global en esta investigación es considerada la sensación de bienestar que expresa el paciente diabético con relación a la salud, tratamiento ordenado, complicaciones y efectos de la enfermedad. La calidad de vida relacionada con la salud, como lo cita Schwartzmann (31), es asumida como un proceso dinámico y cambiante que incluye interacciones continuas entre el paciente y su medio ambiente. De acuerdo con este concepto, la calidad de vida en una persona enferma es la resultante de la interacción del tipo de enfermedad y su evolución, la personalidad del paciente, el grado de cambio que inevitablemente se produce en su vida, el apoyo social recibido y percibido y la etapa de la vida en que se produce la enfermedad. Los parámetros que determinan la calidad de vida en el diabético tipo 2 se relacionan con:

[...] la sensación de ser un enfermo crónico, variaciones en el estilo de vida derivados del esquema terapéutico, cortejo sintomático derivado del mal control metabólico, complicaciones crónicas microangiopáticas y vasculares, discapacidad física secundaria a amputaciones o secuelas de complicaciones clásicas, deterioro cognitivo, trastornos afectivos y cambios en el entorno social. (32)

La presencia de una enfermedad crónica, considerada por la Organización Mundial de la Salud "como un trastorno orgánico funcional que obliga a una modificación de los estilos de vida de una persona y que tiende a persistir a lo largo de su vida" (33), se constituye en un factor influyente 
en la manera como el paciente percibe la calidad de vida. Esta última, en personas con enfermedades crónicas, se ve afectada debido a que las condiciones de salud constituyen problemas que persisten en el tiempo. El ser diagnosticado con una afección de este tipo acarrea un sinnúmero de implicaciones en los aspectos psicológico, físico, espiritual y social. En el caso de los pacientes diabéticos tipo 2, se puede afectar profundamente la calidad de vida, en términos de bienestar físico, psicológico y social.

La inadecuada calidad de vida del paciente diabético se puede mejorar mediante la educación del equipo de salud y, específicamente, del personal de enfermería. Medina (17), en el libro Ciencia y disciplina de enfermería, considera que "la educación sanitaria es una de las principales funciones del personal de enfermería, proceso en el que se deberá tener en cuenta la percepción de cada paciente antes de dar información cognitiva, permitiéndole trabajar en el mismo cuadro conceptual que el paciente". De igual modo, Watson (18), en la Filosofía y teoría del cuidado, plantea que uno de los diez factores cuidativos que la enfermera(o) debe fortalecer o desarrollar "hace referencia a promocionar la enseñanza y el aprendizaje interpersonal. Mediante este factor el personal de enfermería puede brindar un cuidado más humano, a su vez que informa al paciente y deriva a este la responsabilidad de su salud y bienestar”. Según Medina (17) y Watson (18), es importante resaltar que mediante acciones de información y educación el personal de enfermería podrá acercarse al paciente y orientarlo en la situación de salud actual que vive, así como en la búsqueda de acciones que favorezcan el autocuidado y la autogestión para obtener mejores condiciones de vida.

\section{Conclusiones}

El bajo nivel de escolaridad afecta la poca información que estos pacientes manejan acerca de su enfermedad, prevención de complicaciones y medidas terapéuticas. La escolaridad en personas con enfermedades crónicas como la diabetes influye para que obtengan información y asuman un rol positivo en el autocuidado que deben iniciar y mantener desde el momento en que conoce el diagnóstico.

La calidad de vida percibida por hombres y mujeres, aunque tiene diferencias minimas, es muy semejante para los dos sexos. Esto indica la posibilidad de fortalecer las acciones de educación en todos los pacientes, a fin de lograr un compromiso favorable hacia el autocuidado, fomentado por la promoción de estilos saludables y la prevención de factores determinantes para la presencia de complicaciones.

La calidad de vida percibida se ve afectada en la subescala de impacto al tratamiento, por la persistencia de síntomas propios de la diabetes, como poliuria, complicaciones cardiovasculares, dificultades para adoptar una dieta balanceada y valoración subjetiva del estado de salud por sentirse enfermo.

La calidad de vida en un paciente crónico es la resultante de la interacción de varios factores, entre ellos la adherencia al tratamiento, el apoyo social recibido y percibido y la percepción personal que tenga el paciente según el contexto cultural. 


\section{Financiación}

Los recursos requeridos para el desarrollo de la investigación se obtienen de recursos propios.

\section{Conflicto de interés}

Para el desarrollo de la investigación se ha cumplido con normas éticas que responda el derecho a la intimidad y confidencialidad, entre otros. No existe conflicto de intereses.

\section{Referencias}

1. Ministerio de la Protección Social. Guía 17: Guía de atención de la diabetes mellitus tipo 2. En Guía de promoción de la salud y prevención de la enfermedad. Bogotá; 2007. p. 361-439.

2. Quirantes A, López L, Curbelo V, Montano J, Machado P, Quirantes A. La calidad de vida del paciente diabético. El Hospital [internet]. Febrero/Marzo 2003 [Citado enero 2011]:36-41. Disponible en http:// bvsms.saude.gov.br/bvs/is_digital/is_0403/pdf/IS23(4)095.pdf

3. Federación Diabetológica de Colombia. Alerta sobre el incremento de la diabetes. Periódico Diabetes Control y Difusión [internet]. 2002;1(1):1.

4. Organización Mundial de la Salud. Situación de salud de las Américas: indicadores básicos. Vol. I. Regional. Washington; 2006.

5. Organización Panamericana de la Salud. Indicadores de Salud Colombia 2002 [internet]. 2002. [Citado septiembre 2005]. Disponible en: http://www.col.ops.oms.org/sivigila/indicadores/indicadores2002.

6. Instituto Departamental de Salud. Sistema de información estadística, primera causas de mortalidad según el CIE-10 2007 [internet]. [Citado marzo de 2010]. Disponible en: http://www.ids.gov.co/index. php?option=com_content\&view $=$ article $\&$ id $=51:$ info $2007 \&$ catid $=1 \&$ Ite $\operatorname{mid}=78$

7. Ramírez M. Contexto sociopolítico y legal que fundamenta la implementación de programas o proyectos para el cuidado de la salud. Revista Ciencia y Cuidado. 2006;3(3):127-39.

8. Organización Mundial de la Salud, Organización Panamericana de la Salud. Iniciativa de diabetes para las Américas (DIA): plan de acción para América latina y el Caribe 2001-2006. Washington; 2001.

9. Valadez I, Aldrete M, Alfaro N. Influencia de la familia en el control metabólico del paciente diabético tipo II. Salud Pública (Méx). 1993;35(5):464-70.

10. Branka L. Enfermedades no trasmisibles en las Américas: construyamos un mundo más saludable [internet]. Washington; 2011 [citado abril de 2010] Disponible en: http://www.paho.org/spanish/ ad/dpc/nc/pan-mtg-07-2cncd-cmn-reg-strat.pdf

11. Ministerio de la Protección Social. Norma técnica para la prevención de enfermedad crónica y mantenimiento de la salud en el individuo sano mayor de 45 años. Bogotá; 2000. 
12. Alleyne Sir G. La diabetes una declaración para las Américas. Sanitaria Panamericana. 2006;121(5):461-6.

13. De los Ríos J, Barrios P, Ávila T. Calidad de vida en pacientes con diabetes mellitus tipo 2 y nefropatía diabética. Medica Hospital General de México. 2005;68(3):142-54.

14. Vinaccia S, Orozco L. Aspectos psicosociales asociados con la calidad de vida de las personas con enfermedades crónicas. Perspectivas en Psicología. 2005;1(2):125-37.

15. Tuesca R. La calidad de vida, su importancia y como medirla. Salud Uninorte. 2005;(21):76-86.

16. Vargas L. Marco para el cuidado de la salud en situaciones de enfermedad crónica. Invest Enferm: Imagen Desarr. 2010;12(1):79-94.

17. Medina J. Ciencia y disciplina de enfermería. México: Interamericana; 2001.

18. Watson J. Filosofía y teoría de los cuidados humanos. 3a ed. s. 1.: Universidad de Colorado; 1999.

19. Zamberlan C, Calvetti A, Deisvaldi J, De Siqueira H. Calidad de vida, salud y enfermería en la perspectiva eco sistémica. Enfermería Globa (Esp). 2010;(20):1-7.

20. Machado A, Anarte M, Ruiz M. Predictores de calidad de vida en pacientes con diabetes mellitus tipo 1. Clínica y Salud [internet]. 2010 [citado julio de 2012];21(1):35-47. Disponible en: http://www. redalyc.org/pdf/1806/180615542004.pdf

21. Millán M. Cuestionario de calidad de vida específico para la diabetes mellitus (EsDQOL). Atención Primaria [internet]. 2002 [citado diciembre 2011];29(8):517-21. Disponible en http://www.elsevier.es

22. Millán M. Validación de un cuestionario de calidad de vida del paciente diabético y material didáctico para la educación en diabetes: dos instrumentos complementarios. [Tesis]. Universidad de Barcelona, España; 1996.

23. Robles R, Cortázar J, Sánchez J, Páez F, Nicolini H. Evaluación de la calidad de vida en diabetes mellitus tipo 2: propiedades psicométricas de la versión en español del DQOL. Psicothema. 2003;15(2):247-52.

24. Antuña de Alaiz R, Hirsch A. Calidad de vida en diabéticos tipo 1: comparación entre multidosis de insulina y bomba externa de insulina [internet]; 2012 [citado julio de 2012]. Disponible en: http:// www.clinidiabet.com/es/nuestraclinica/index.htm

25. González M, Rivas V. Calidad de vida de los adultos con diabetes mellitus tipo 2 [internet]; 2008 [citado enero de 2011]. Disponible en: http://www.archivos.ujat.mx/dip/divulgacion\%20y\%20video\%20cinetifico\%202008/DACS/VRivasA\%201.pdf

26. Gualdrón J. Factores básicos condicionantes del auto cuidado asociados a la capacidad potencial para auto cuidarse y promover la salud en personas de 18 años y más que viven en situación de enfermedad cardiovascular y asistieron a la consulta externa de cardiología del Hospital Nacional Rosales. Documento procedente del I Foro Regional de Investigación; El Salvador; 2003. 
27. Salazar J, Colunga C, Pando M, Aranda C, Torres T. Diabetes y calidad de vida: estudio comparativo en adultos con y sin diagnóstico de diabetes tipo 2 atendidos en centros de salud en Guadalajara. Revista Investigación en Salud (Mex). 2007;IX(2):88-93.

28. Concha M, Rodríguez C. Funcionalidad familiar en pacientes diabéticos e hipertensos compensados y descompensados. Theoria. 2010;19(1):41-50.

29. Cárdenas V, Pedraza C, Lerma R. Calidad de vida del paciente con diabetes mellitus tipo 2. Ciencia (UANL). 2005;VIII(3):351-7.

30. Gervas M. Calidad de vida y automanejo de la enfermedad en diabéticos insulinodependientes de valladolid [tesis]. Universidad de Valladolid, España; 1997.

31. Schwartzmann L. Calidad de vida relacionada con la salud: aspectos conceptuales. Cienc Enferm [internet]. 2003 [citado 15 de julio de 2012];9(2):9-21. Disponible en: http://www.scielo.cl/scielo. php?pid=S0717-95532003000200002\&script=sci_arttext

32. De Pablos P. La calidad de vida se resiente en los pacientes con diabetes. Hospital Universitario Dr. Negrín, Las Palmas de Gran Canaria. Reesmgraf [internet]. 2006 [citado enero de 2010];7(7):179. Disponible en: http://www.economiadelasalud.com/ediciones/72/08_pdf/analisiscalidaddevida.pdf

33. Romeo E, Acosta S, Carmona I, Jaimes A, Masco M, Áez A et al. Calidad de vida de personas con diabetes mellitus 2 en Cartagena Colombia. Ciencias Biomédicas [internet]. 2010 [citado enero de 2011];1(10):190-8. Disponible en: http://www.unicartagena.edu.co/ medicina/09\%20CALIDAD \%20DE \%20VIDA \%20DIABETES.pdf 